\title{
Les relations entre communautés linguistiques en contexte scolaire et communautaire : regards croisés sur Montréal et Bruxelles
}

\section{Relationships Between Linguistic Communities in a Scholastic and Community Context: Comparison of Montreal and Brussels \\ Las relaciones entre comunidades lingüísticas en contexto escolar y comunitario: una comparación entre Montreal y Bruselas}

\section{Benoit Côté et Laurence Mettewie}

Volume 36, numéro 1, printemps 2008

Rapports ethniques et éducation : perspectives nationales et internationales

URI : https://id.erudit.org/iderudit/018087ar

DOI : https://doi.org/10.7202/018087ar

Aller au sommaire du numéro

\section{Éditeur(s)}

Association canadienne d'éducation de langue française

ISSN

0849-1089 (imprimé)

1916-8659 (numérique)

Découvrir la revue

Citer cet article

Côté, B. \& Mettewie, L. (2008). Les relations entre communautés linguistiques en contexte scolaire et communautaire : regards croisés sur Montréal et Bruxelles. Éducation et francophonie, 36(1), 5-24.

https://doi.org/10.7202/018087ar

\section{Résumé de l'article}

L'objectif de cet article est de présenter et de comparer le rôle des milieux communautaires et scolaires dans les rapports entre communautés linguistiques historiques de Montréal et de Bruxelles. Même si ces deux contextes linguistiques sont différents à bien des égards, ils partagent deux caractéristiques qui laissent croire que l'une et l'autre des communautés puissent apprendre de l'expérience qu'elles ont développée en parallèle, soit : un contexte d'ambiguïté de dominance ethnique et un système scolaire divisé linguistiquement. Sur base de recherches récentes, nous ferons le bilan de l'état des rapports dans les milieux scolaires et communautaires entre francophones et anglophones montréalais d'une part (Pagé et al. 2007, Côté 2005) et entre jeunes francophones et néerlandophones à Bruxelles d'autre part (Mettewie 2004, Janssens et Van Mensel 2006). L'impact de ce contact intercommunautaire sur les représentations et les attitudes fera émerger, en conclusion, des questions qui, à la lecture de ces deux contextes, induisent des pistes de recherches complémentaires et comparatives.
Tous droits réservés (C) Association canadienne d'éducation de langue française, 2008 cécument est protége par la loi sur le droit d'auteur. L'utilisation des services d’Érudit (y compris la reproduction) est assujettie à sa politique d'utilisation que vous pouvez consulter en ligne. 


\section{Les relations entre communautés linguistiques en contexte scolaire et communautaire : regards croisés sur Montréal et Bruxelles}

\section{Benoit CôTÉ}

Professeur adjoint, Département de psychologie, Université de Sherbrooke, Québec, Canada

\section{Laurence METTEWIE}

Chargée de cours, Département de langues et littératures germaniques, Université de Namur (FUNDP), Wallonie, Belgique

\section{RÉSUMÉ}

L'objectif de cet article est de présenter et de comparer le rôle des milieux communautaires et scolaires dans les rapports entre communautés linguistiques historiques de Montréal et de Bruxelles. Même si ces deux contextes linguistiques sont différents à bien des égards, ils partagent deux caractéristiques qui laissent croire que l'une et l'autre des communautés puissent apprendre de l'expérience qu'elles ont développée en parallèle, soit : un contexte d'ambiguïté de dominance ethnique et un système scolaire divisé linguistiquement. Sur base de recherches récentes, nous ferons le bilan de l'état des rapports dans les milieux scolaires et communautaires 
entre francophones et anglophones montréalais d'une part (Pagé et al. 2007, Côté 2005) et entre jeunes francophones et néerlandophones à Bruxelles d'autre part (Mettewie 2004, Janssens et Van Mensel 2006). L'impact de ce contact intercommunautaire sur les représentations et les attitudes fera émerger, en conclusion, des questions qui, à la lecture de ces deux contextes, induisent des pistes de recherches complémentaires et comparatives.

\section{ABSTRACT}

\section{Relationships Between Linguistic Communities in a Scholastic and Community Context: Comparison of Montreal and Brussels}

Benoit CôTÉ

University of Sherbrooke, Quebec, Canada

Laurence METTEWIE

University of Namur (FUNDP), Belgium

The objective of this article is to present and compare the role of community and school milieus through relationships between the historical linguistic communities of Montréal and Brussels. Although these two linguistic contexts differ in many ways, they share two characteristics, which suggest that each of these communities could learn from the experience they developed in parallel - a context of the ambiguity of ethnic dominance and a linguistically divided school system. On the basis of recent research, we will examine the connections in the school and community milieus between French- and English-speaking Montréalers (Pagé et al. 2007, Côté 2005) and between young French-speaking and Dutch-speaking students (Mettewie 2004, Janssens and Van Mensel 2006). In conclusion, the impact this intercommunity contact has on representations and attitudes brings out questions which, in examining these two contexts, reveals possibilities for complimentary and comparative research. 


\section{RESUMEN}

\section{Las relaciones entre comunidades lingüísticas en contexto escolar y comunitario: una comparación entre Montreal y Bruselas}

Benoit CÔTÉ

Universidad de Sherbrooke, Quebec, Canadá

Laurence METTEWIE

Universidad de Namur (FUNDP), Bélgica

El objetivo de este artículo es presentar y comparar el rol de los medios comunitarios y escolares en las relaciones entre comunidades lingüísticas históricas en Montreal y en Bruselas. Aunque dichos contextos lingüísticos difieran en muchos aspectos, comparten dos características que permiten pensar que ambas comunidades puedan aprender de la experiencia que de manera paralela han vivido, es decir: un contexto de ambigüedad en el campo étnico y un sistema escolar lingüísticamente dividido. Basándonos en investigaciones recientes, realizaremos un balance de las relaciones en el medio escolar y comunitario entre francófonos y anglófonos en Montreal (Pagé et al, 2007; Coté 2005) y entre los jóvenes francófonos y neerlandeses en Bruselas (Mattewie 2004; Janssens y Van Mensel 2006). El impacto del contacto ínter-comunitario sobre las representaciones y las actitudes mostrará, en conclusión, cuestiones que de acuerdo con la lectura de dichos contextos, sugieren pistas complementarias y comparativas de investigación.

\section{Montréal et Bruxelles : semblables et différentes}

Chaque société doit, d'une manière ou d'une autre, gérer les relations entre les divers groupes ethniques, religieux ou linguistiques qui évoluent en son sein. Si certaines situations sont plus comparables, en fonction par exemple de la quantité et des sources d'immigration qui viennent complexifier ces relations, d'autres sont très différentes. Bien entendu, les sociétés belge et québécoise se distinguent notamment par leur histoire, leur environnement géographique ou leurs structures politiques. Cependant, elles partagent également deux caractéristiques fondamentales: un contexte d'ambiguïté quant à la dominance ethnique et au système scolaire divisé linguistiquement. Celles-ci rendent intéressante une comparaison des relations entre les membres des deux communautés linguistiques historiques de leur environnement respectif, soit francophone et anglophone du Québec et néerlandophone et francophone de Belgique. L'objectif central de cet article est donc de présenter et comparer le rôle des milieux communautaires et scolaires dans les rapports entre ces communautés linguistiques historiques de Montréal et de Bruxelles. 


\section{Dominances ethniques ambiguës}

Tout d'abord, il faut mentionner que, contrairement à d'autres sociétés comme la France ou l'Angleterre où un groupe linguistique ou ethnique est clairement dominant, la Belgique et le Québec sont caractérisés par une ambiguïté de dominance ethnique, c'est-à-dire par une difficulté à identifier de manière consensuelle le groupe majoritaire (McAndrew \& Gagnon, 2000). Même si au Canada les francophones sont minoritaires avec seulement $22 \%$ de la population, comparativement aux $58 \%$ d'anglophones, l'effet est inverse au Québec, où les anglophones sont minoritaires et représentent environ $8 \%$ de la population, versus $81 \%$ de francophones. Montréal, quant à elle, se caractérise comme l'une des grandes villes canadiennes, avec Toronto, Ottawa et Vancouver, ayant une grande diversité linguistique, soit avec une proportion de $68 \%$ de francophones, $12 \%$ d'anglophones et $19 \%$ d'allophones (Statistique Canada, 2001). Historiquement, les communautés anglophones se sont établies davantage dans l'ouest de l'île, tandis que la communauté francophone occupait davantage l'est. De nos jours, même si l'est de l'île de Montréal reste encore très francophone et l'ouest garde une forte dominance anglophone, cette division n'est plus aussi étanche et l'on retrouve des francophones dans tous les secteurs de l'île (Archambault, Ray, Rose \& Séguin, 2007).

Figure 1 : Répartition de la population selon la langue maternelle par secteurs de recensement, Communauté urbaine de Montréal, 1996*

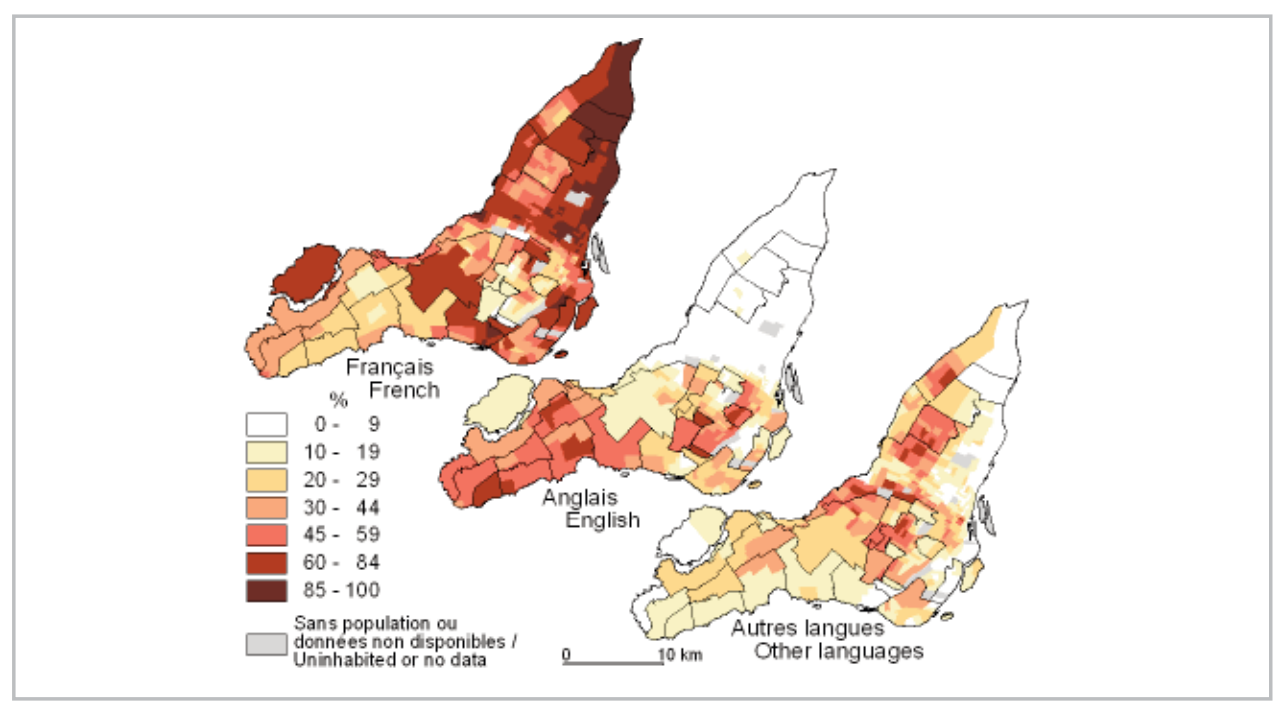

" Image tirée de: Atlas: Immigration et Métropoles de Archambault, Ray, Rose \& Séguin, site web consulté le 10 octobre 2007: http://im.metropolis.net/research-policy/research_content/atlas/carteA2.html.

La situation belge est au moins aussi complexe du point de vue des dominances des groupes linguistiques que la situation canadienne. Les trois premiers articles de la constitution belge dans sa version de 1994 (voir www.senate.be) stipulent ainsi que 
la Belgique est un État fédéral composé de trois communautés (Communauté française, flamande et germanophone) et de trois régions (Région wallonne, flamande et bruxelloise), qui par malheur pour le lecteur, ne coïncident ni géographiquement, ni linguistiquement. Basé sur le principe de territorialité, l'article 4 de cette constitution précise, en effet, qu'il existe quatre régions linguistiques: la région de langue française, celle de langue néerlandaise, la région bilingue (français/néerlandais) de Bruxelles-Capitale et la région de langue allemande. Les statistiques indiquent que démographiquement, la région flamande est largement dominante avec $57,8 \%$ de la population (données 2006 de la Direction générale de Statistique et Information économique, voir www.statbel.be) contre $32,5 \%$ pour la région wallonne et 9,7\% pour la région bruxelloise. Mais ces chiffres ne disent rien quant au profil linguistique de la population elle-même, puisque depuis 1947 les recensements linguistiques ne sont plus tolérés. Si d'expérience on peut admettre que les régions flamande et wallonne (hors communauté germanophone) sont linguistiquement assez homogènes, on peut donc admettre que les néerlandophones sont démographiquement plus nombreux que les francophones. Cependant, pour la région bilingue de Bruxelles, la question du profil linguistique ne peut être abordée que par des enquêtes avec des échantillonnages représentatifs. Par ailleurs, le statut d'une part bilingue de la région et d'autre part international de la ville elle-même, hôte d'institutions européennes, de l'OTAN, d'entreprises internationales, mais aussi d'une population migrante importante, implique une diversification des profils linguistiques qui dépassent largement une polarisation francophones vs néerlandophones. Ainsi Janssens (2007) constate-t-il que dans la région de Bruxelles-Capitale, ce sont les francophones qui sont majoritaires avec plus de $57 \%$ de la population, pour $7 \%$ de néerlandophones. Mais il faut ajouter à cela un peu moins de $10 \%$ de bilingues français/néerlandais, $11 \%$ de "nouveaux" bilingues (français/langue d'émigration) et enfin, $16 \%$ d'allophones. Les néerlandophones sont donc à la fois majoritaires au niveau national et minoritaires à Bruxelles, même si leur représentativité politique et leurs droits linguistiques y sont assurés. Enfin, il faut noter que si les néerlandophones de la capitale se trouvent principalement au nord de la ville, la gentrification du centre de Bruxelles, assortie d'une politique culturelle très active de la Communauté flamande, a permis le développement d'un noyau néerlandophone très huppé au cœur de la ville, bordé de quartiers administratifs ou paupérisés. À Bruxelles, même si les profils linguistiques sont très diversifiés, on peut néanmoins conclure que s'il est question de francophones et de néerlandophones, ceux-ci ne vivent pas les uns avec les autres, mais bien les uns à côté des autres. 
Figure 2 : Répartition des langues officielles dans les trois régions en Belgique

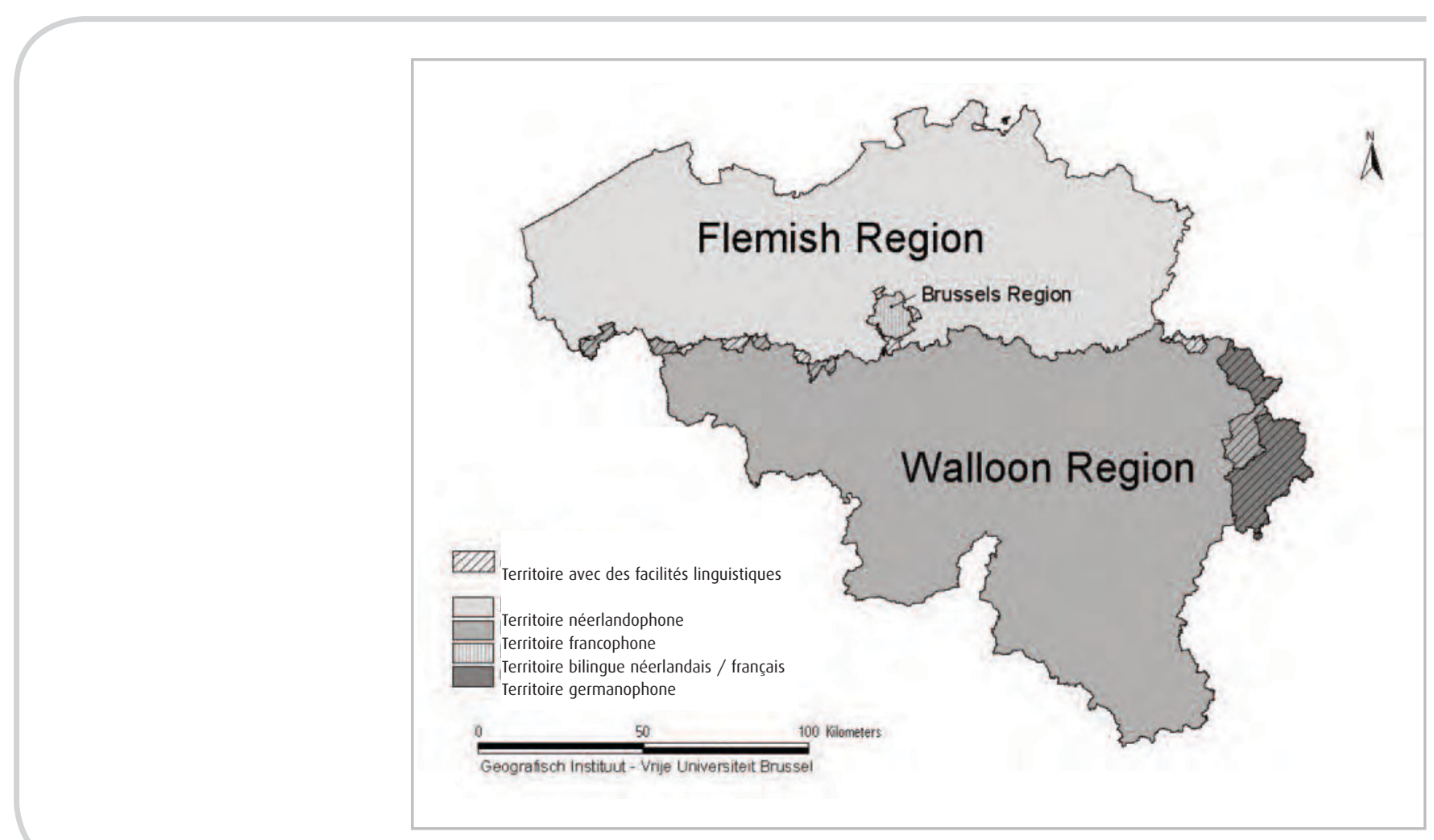

\section{Possibilités de contacts dans des systèmes scolaires encadrant les frontières linguistiques}

Dès lors, ce contexte historique d'ambiguïté amène des défis particuliers dans les rapports entre les deux communautés linguistiques, qui avec des systèmes scolaires encadrés par les frontières linguistiques, ne peuvent compter sur une scolarisation commune pour développer ou renforcer les bases d'un tissu social commun. En effet, contrairement à d'autres contextes nationaux, comme celui des États-Unis où dans les années 50 la Cour Suprême a déclaré anticonstitutionnelle la ségrégation scolaire en écoles de «blancs » et de «noirs» (Brown v. Board of Education, 1954: voir Brewer \& Miller, 1996), les contextes québécois et belge, malgré quelques voies d'exception, qui ne seront pas le propos principal ici, utilisent des systèmes scolaires linguistiquement séparés. Cette stratégie, dont l'un des buts est évidemment d'assurer la pérennité des groupes linguistiques en compétition, ne permet que très peu l'utilisation du système d'éducation pour rapprocher les communautés linguistiques de ces sociétés divisées.

\section{Le contexte québécois}

Dans le contexte québécois, c'est la loi 101 qui, depuis 1977, rend obligatoire la scolarisation en français aux niveaux primaire et secondaire, des francophones et des 
allophones, ne permettant l'accès aux écoles anglaises uniquement à la minorité historique anglophone. Dans ce contexte de cloisonnement scolaire, et ce en dépit des efforts du ministère de l'Éducation, des Loisirs et du Sport (MELS) pour financer les échanges linguistiques entre écoles francophones et anglophones québécoises, les contacts entre ces communautés, soutenus par le système scolaire, restent marginaux (Côté, 2007). Cependant, malgré ce constat, les recherches récentes rapportent que les contacts entre les jeunes de ces deux groupes linguistiques, sur l'île de Montréal, sont très fréquents dans le milieu communautaire (Pagé, Côté \& Lasry, 2007). Plus précisément, ils se rencontrent lors d'activités sportives, culturelles ou sociales soutenues par des organismes de loisirs à but non-lucratif, généralement subventionnés par les différents paliers de gouvernement.

Les niveaux collégial ${ }^{1}$ et universitaire, quant à eux, ne sont pas soumis à de telles règles et permettent donc un libre choix par rapport à la langue d'enseignement. Malgré le fait que l'on retrouve une proportion extrêmement faible d'étudiants anglophones dans les collèges francophones, même à Montréal, les francophones quant à eux représentent jusqu'à $25 \%$ ou $30 \%$ de la population dans les collèges anglophones de Montréal et la proportion peut être plus élevée encore dans les collèges anglophones à l'extérieur de la métropole (Côté, 2005). Ces contextes scolaires sont donc très riches en possibilités de contacts entre ces deux communautés linguistiques.

\section{Le contexte belge}

En Belgique, ce sont les Communautés qui sont chargées d'organiser, de gérer et de financer l'enseignement, à l'intérieur d'un cadre législatif très strict en matière de langues d'enseignement et d'enseignement des langues. Ainsi, la langue d'enseignement se doit d'être la langue de la région, c'est-à-dire le néerlandais en région flamande et le français en région wallonne (à l'exception des écoles de la Communauté germanophone, que nous n'aborderons pas ici). Dans la région bilingue de Bruxelles-Capitale, tant la Communauté française que la Communauté flamande sont compétentes en matière d'enseignement. Ce qui signifie qu'à Bruxelles, il existe deux systèmes d'enseignement totalement indépendants et parallèles, l'un en français, l'autre en néerlandais, et ce, des classes maternelles à l'université. Un enseignement bilingue co-organisé par les deux Communautés, de type bidirectionnel (appelé dual language education, en anglais) est donc impossible tant d'un point de vue politique que légal. Or, dans une ville comme Bruxelles, les besoins en langues sont prégnants et l'enseignement de la langue seconde en Communauté française ne parvient pas à répondre aux exigences du marché de l'emploi (Mettewie, 2004; Mettewie, Van Mensel, \& Belang, 2006). Si depuis 1998, l'enseignement en immersion (de type EMILE / CLIL ${ }^{2}$ ) est autorisé dans les écoles de

1. Dans le système scolaire québécois, le niveau collégial, généralement appelé CÉGEP, est le niveau d'études de 2 ou 3 ans que l'on retrouve entre le niveau secondaire et le niveau universitaire. Généralement, les formations préuniversitaires sont d'une durée de 2 ans et les formations techniques sont d'une durée de 3 ans. Les étudiantes et étudiants de ce niveau ont généralement entre 17 et 19 ans.

2. Enseignement d'une matière par intégration d'une langue étrangère (EMILE) ou Content and Language Integrated Learning (CLIL), voir www.clilcompendium.com ou www.euroclic.net. 
la Communauté française, sous des conditions très strictes, il n'est guère développé à Bruxelles (5\% à $10 \%$ des écoles francophones de la capitale proposaient ce type d'enseignement en 2006, voir www.enseignement.be). Outre des problèmes organisationnels, l'enseignement en immersion a comme concurrent direct l'enseignement néerlandophone à Bruxelles. En effet, contrairement aux autres régions, les parents bruxellois jouissent de la «liberté du père de famille» et depuis les années 1980 de plus en plus de parents francophones de la capitale choissent d'inscrire leurs enfants dans les écoles de la Communauté flamande. Ceci permet à l'enseignement néerlandophone d'augmenter ses parts de marché dans l'enseignement à Bruxelles (en moyenne 16,7 \% de la population scolaire à Bruxellles en 2000, pour moins de $10 \%$ de néerlandophones dans la capitale; Mettewie, 2004). Les statistiques au $1^{\mathrm{er}}$ février 2007 (www.vgc.be/onderwijs/) indiquent que l'enseignement maternel (de 3 à 6 ans) accueille $10 \%$ d'enfants néerlandophones et $20 \%$ d'enfants bilingues français/néerlandais, pour $29 \%$ de francophones et $41 \%$ d'allophones. Même si la proportion d'élèves néerlandophones est plus importante aux niveaux primaire et secondaire ( $14 \%$ et $34 \%$ de néerlandophones et $21 \%$ et $28 \%$ de bilingues), la présence d'élèves ne parlant pas le néerlandais à la maison reste très importante dans ces écoles néerlandophones de la capitale. Nous n'aborderons pas ici les motivations, ni les implications pédagogiques ou de politique linguistique que cela implique (voir Mettewie, 2008), mais bien la situation particulière de contact que cela engendre. En effet, les écoles néerlandophones de Bruxelles sont un des rares lieux où des membres des deux principales communautés linguistiques se côtoient quotidiennement et grandissent ensemble. C'est le principal cas de contact intergroupe dans une société belge de plus de plus polarisée.

Il apparaît donc que les contacts entre communautés linguistiques historiques à Montréal et à Bruxelles soient loin d'être institutionnalisés et dépassent les frontières linguistiques imposées par les systèmes scolaires.

\section{L'impact des milieux scolaires et communautaires sur les rapports intercommunautaires}

Il apparaît donc que les contacts entre communautés linguistiques historiques à Montréal et à Bruxelles soient loin d'être institutionnalisés et dépassent les frontières linguistiques imposées par les systèmes scolaires. Il est donc intéressant d'analyser l'impact que ces contacts peuvent avoir sur les rapports intergroupes des jeunes citadins.

Si les méthodologies des études présentées dans cette contribution diffèrent, les objectifs et les résultats quant à eux convergent. Nous présenterons donc en parallèle l'analyse de la situation à Montréal et à Bruxelles et conclurons par une comparaison des résultats et des questionnements qu'ils impliquent.

\section{Le portrait montréalais}

Deux recherches récentes, soit celle de Pagé et ses collaborateurs (2007) et celle de Côté (2005), permettent de mieux saisir l'état actuel des rapports entre francophones et anglophones dans les milieux scolaires et communautaires montréalais. 
Dans un premier temps, Pagé et ses collaborateurs (2007) ont effectué une recherche auprès de 1109 jeunes allant dans des écoles secondaires francophones de l'île de Montréal, afin d'étudier leur participation à des activités parascolaires et communautaires avec des membres de l'autre communauté linguistique. Cette recherche décrit, entre autres, l'identité de ces jeunes, leurs usages linguistiques, leurs attitudes par rapport à l'autre groupe linguistique, la description de la qualité et de la quantité des contacts qu'ils ont avec les membres de l'autre groupe linguistique ainsi que leur perception de l'impact de leur participation à de telles activités.

\section{Contacts entre jeunes francophones et anglophones en milieu communautaire} à Montréal

Un premier constat, surprenant en termes d'ampleur non anticipée, veut que malgré le fait que le système scolaire québécois soit divisé et ne favorise que peu les échanges entre écoles francophones et anglophones, les jeunes qui participent à des activités sportives et culturelles avec des membres de l'autre communauté linguistique sont près d'être trois fois plus nombreux que ceux qui n'y participent pas (813 vs 296). Du côté est de l'île, dans les quartiers de Montréal où la population est majoritairement francophone, $47,5 \%$ des élèves déclarent participer à ces activités communautaires mixtes, de même que dans l'ouest de l'île, où la population est davantage mixte linguistiquement, mais avec une nette prédominance anglophone, entre $74,5 \%$ et $81,9 \%$ des élèves (selon les écoles) participent à ces activités mixtes. De plus, les résultats montrent que la participation à ces activités n'est ni épisodique, ni sporadique, car les jeunes s'y investissent sur une période variant d'un an à quatre ans et la très grande majorité de ceux-ci déclarent y consacrer passablement beaucoup de temps.

La recherche de Pagé et ses collaborateurs (2007) met également en lumière que les contacts informels entre ces jeunes sont très fréquents en dehors de ces activités communautaires. Dans l'ouest de l'île, la très grande majorité des jeunes francophones disent avoir dans leurs réseaux plusieurs amis anglophones et plus de la moitié des répondants de l'est de l'île affirment la même chose. De plus, il ne faut pas oublier que plusieurs allophones (Québécois de $1^{\text {re }}$ et $2^{\mathrm{e}}$ générations ${ }^{3}$ ) allant à l'école française habitent dans l'ouest de l'île, ce qui rend ces contacts linguistiques plus complexes qu'une simple dichotomie «francophones» versus «anglophones». En fait, dans l'ouest de Montréal, $16 \%$ à $30 \%$ de ceux qui fréquentent l'école française affirment utiliser davantage l'anglais que le français en dehors de l'école et, évidemment, la majorité d'entre eux sont des Québécois allophones issus de l'immigration récente. Toutes ces données dépeignent donc Montréal comme un lieu plus riche en termes de fréquence de rencontres linguistiques réelles pour les jeunes de niveau secondaire, que l'on aurait pu croire avec des systèmes scolaires divisés.

3. Nous entendons ici que les personnes qui sont nées à l'extérieur du Québec, qui sont devenues citoyens canadiens et qui vivent dans la province de Québec, sont des "Québécois de première génération ». Leurs enfants, quant à eux, sont des « Québécois de deuxième génération ». 
Les données recueillies sur ces jeunes qui participent à ces activités communautaires sportives et culturelles linguistiquement mixtes, montrent que tant les francophones que les anglophones ont plus de contacts informels (dans les parcs, les rues, les commerces, etc.) avec des membres de l'autre groupe linguistique, dans leur vie de tous les jours, qu'en ont ceux qui ne participent pas à ces activités. Dans le même sens, ceux qui participent aux activités mixtes utilisent plus souvent la langue de l'autre communauté linguistique avec leurs amis et les membres de leur famille, que ceux qui ne participent pas à de telles activités.

On observe également que les francophones qui participent à ces activités mixtes, qu'ils soient d'ascendance ancienne ou d'origine immigrante, s'évaluent plus compétents dans la langue anglaise que s'estiment les francophones qui ne sont pas engagés dans ces activités. Toutefois, le constat n'est pas le même pour les jeunes anglophones. Dans leur cas, les données montrent que les anglophones, peu importe s'ils participent ou non à des activités mixtes, s'évaluent plus compétents dans l'autre langue (donc en français) que ne le font les francophones (quant à la langue anglaise). Un constat semblable a été observé lors d'un projet pilote, intitulé $R A P$ Montréal et financé par Canadian Parents for French et le ministère de l'Éducation, des Loisirs et du Sport du Québec. Il s'agit d'un rapprochement linguistique entre deux classes de langue seconde d'écoles secondaires de l'ouest de l'île de Montréal, l'une francophone et l'autre anglophone. En effet, le comité organisateur du RAP Montréal a constaté que les élèves francophones semblaient moins confiants en leur compétence dans leur langue seconde que ne l'étaient les élèves anglophones. L'hypothèse principale de cet écart de perception dans l'auto-évaluation de leur compétence linguistique, qui devra être vérifiée par une analyse plus approfondie, serait liée à deux facteurs favorisant la maîtrise du français. Il s'agit, d'une part, de la qualité des cours de français offerts dans le système anglophone, où l'on retrouve fréquemment des stratégies d'immersion, et d'autre part, de l'omniprésence de la langue française à Montréal (due à la loi 101), qui exposerait potentiellement davantage les jeunes anglophones à la langue française que l'inverse.

\section{L'impact du rapprochement intergroupe dans le milieu communautaire à Montréal}

Dans un autre ordre d'idées, il semble que le milieu communautaire offre un contexte adéquat pour le rapprochement intercommunautaire. La théorie des contacts intergroupes, dont les bases ont été fondées par Allport (1954), stipule grosso modo que sous certaines conditions favorables, plus il y aura de contacts entre les membres de groupes différents, plus ils vont apprendre à se connaître et moins il y aura de discrimination entre eux (Côté, 2005; Gaertner, Rust, Dovidio, Bachman \& Anastasio, 1994; Taylor, Dubé \& Bellerose, 1986; Hewstone \& Brown, 1986). Ceci signifie que tous les contextes de contacts intergroupes n'amèneront pas automatiquement des effets positifs. Si les conditions essentielles ne sont pas présentes, il est donc possible que ces rencontres ne produisent aucun effet, ou encore créent des effets pervers sur les perceptions et attitudes face à l'exogroupe. Évidemment, la liste des conditions favorables ou nécessaires aux contacts intergroupes n'a cessé de s'allonger dans la 
deuxième moitié du $20^{\mathrm{e}}$ siècle et des auteurs comme Pettigrew (1998) et Dovidio et ses collaborateurs (2003) ont effectué d'excellentes synthèses de ces conditions, que nous reprendrons plus loin.

La recherche de Pagé et ses collaborateurs (2007) mentionne que les activités communautaires sont perçues par les jeunes Montréalais comme respectant les grands principes de la théorie des contacts, c'est-à-dire que 1) la coopération entre les membres des deux communautés linguistiques est plus forte que la compétition, et ce même s'il est logique de s'attendre à un certain degré de compétition dans les activités sportives; 2) le niveau d'interaction entre les francophones et les anglophones est élevé; 3 ) ils sentent que les personnes qui détiennent une autorité (parents et animateurs) soutiennent explicitement des normes favorables au rapprochement intergroupe; et finalement, 4) les statuts des deux langues et des deux communautés linguistiques sont égaux. Dans le même ordre d'idées, la recherche montre que ceux qui participent à ces activités utilisent très fortement un processus de supracatégorisation, c'est-à-dire un processus cognitif qui permet d'associer dans une même catégorie « rassembleuse » des personnes généralement divisées en catégories différentes, par exemple être tous «membres de l'équipe $\mathrm{X}$ » de telle activité sportive, plutôt que des sous-groupes «d'anglophones" et "de francophones".

Enfin, les analyses de Pagé et ses collaborateurs (2007) montrent que la participation à ces activités communautaires mixtes est liée significativement à une plus grande estime de l'autre groupe linguistique $(\mathrm{p}<, 000)$, à une plus grande identification à l'autre groupe $(\mathrm{p}<, 000)$, c'est-à-dire le sentiment de partager des caractéristiques semblables, et à un plus grand désir d'avoir de futurs contacts avec les membres de l'exogroupe $(\mathrm{p}<, 000)$. Évidemment, ces résultats doivent être interprétés comme des corrélations et donc on ne peut pas dire que les activités de contacts sont "la cause " de ces observations, car il est bien possible que ce soit la présence de ces caractéristiques chez ces jeunes qui les amènent à participer aux activités mixtes. Seul un protocole de recherche longitudinal permettrait de mieux distinguer la direction de ces relations.

\section{Qualité et impact des contacts entre jeunes francophones et anglophones en milieu scolaire à Montréal}

Dans un second temps, Côté (2005) a effectué une enquête auprès de 522 étudiants de quatre institutions collégiales anglophones (dont trois dans la région urbaine montréalaise) afin de créer un modèle prédictif de la quantité et de la qualité des contacts interlinguistiques au sein de celles-ci. Rappelons que le niveau collégial est le premier contexte scolaire où la loi 101 ne régit pas dans quelle langue on doit étudier et où il y a donc un libre choix de la langue d'enseignement. Le niveau collégial rend ainsi accessible une première occasion de scolarisation commune entre francophones et anglophones. Ces choix individuels amènent dès lors les institutions anglophones à être très mixtes en termes de groupes linguistiques, avec une proportion élevée de francophones et d'allophones.

Cette recherche démontre, tout d'abord, que les collégiens estiment que les conditions favorables aux contacts intergroupes linguistiques sont fortement 


\section{À la lumière des résultats de la recherche de Côté (2005), l'augmentation du nombre et de la qualité des contacts avec les membres de l'autre communauté linguis- tique ne mène pas pour autant à une assimi- lation à ce groupe.}

présentes dans leurs institutions scolaires, soit 1) que l'interdépendance entre francophones et anglophones est élevée 2) que les collèges (cégeps) anglophones affichent, par l'entremise des membres de la direction et du corps professoral, des normes favorables aux contacts intergroupes et 3) qu'ils sentent une égalité des statuts entre francophones et anglophones. Il ne faut pas croire pour autant que tous les collégiens vivent leurs contacts interlinguistiques de manière idéale, mais bien que même si l'on retrouve tout le spectre d'expérience de contacts, de la plus négative à la plus positive, globalement la moyenne est positive.

La théorie des contacts intergroupes a donc pu être confirmée dans le contexte des collèges anglophones, c'est-à-dire que plus la présence des conditions essentielles est perçue par les répondants : 1) plus il y a une quantité de contacts, mesurée en termes d'amis, de fréquence d'utilisation de l'autre langue, du nombre d'heures partagées en classe avec des étudiants de l'autre langue; 2) plus il y a une qualité de contact, c'est-à-dire une profondeur dans la relation et une aisance (inverse de l'anxiété) lors des contacts avec des membres de l'autre groupe et 3) plus il y a une proximité sociale avec les membres de l'autre groupe linguistique, donc une identification à l'autre groupe et un désir de futurs contacts avec eux.

Toutefois, cette recherche met en lumière une particularité du contexte linguistique, qu'il faut ajouter à la théorie des contacts pour pouvoir l'utiliser adéquatement dans un environnement comme les institutions scolaires montréalaises, à savoir l'auto-évaluation que font les collégiens de leur propre compétence linguistique dans leur langue seconde. Cette auto-évaluation est l'élément qui a le plus d'impact dans la prédiction de la quantité et qualité des contacts que ces jeunes vont avoir avec les membres de l'autre groupe linguistique. Évidemment, l'hypothèse centrale est que le sentiment de compétence dans sa langue seconde est fondamental dans l'apparition ou non de l'anxiété dans les rapports intergroupes, variable largement reconnue pour nuire aux contacts (Clément, Noels \& Denault, 2001; Islam \& Hewstone, 1993; Wilder \& Shapiro, 1989; Stephan \& Stephan, 1985).

Par ailleurs, à la lumière des résultats de la recherche de Côté (2005), l'augmentation du nombre et de la qualité des contacts avec les membres de l'autre communauté linguistique ne mène pas pour autant à une assimilation à ce groupe. En effet, chacun garde une préférence pour son groupe linguistique d'origine, tant en termes d'usage de la langue que du nombre d'amis dans le réseau social.

Finalement, Côté (2005) observe que dans le contexte des collèges anglophones, les rapports entre francophones et anglophones ont lieu dans les faits entre individus qui partagent tous à un certain degré un bilinguisme, allant d'une faible maîtrise de la langue seconde à une excellente maîtrise de celle-ci. Cela complexifie le modèle classique de la théorie des contacts où généralement l'appartenance à un groupe est relativement claire (par exemple " noirs » vs «blancs ", « catholiques » vs «protestants », «juifs» vs «musulmans») ou encore que l'appartenance aux deux groupes est quasi impossible, par exemple il est certainement plus difficile d'être à la fois catholique et protestant à Belfast, qu'il ne l'est d'être à la fois francophone et anglophone à Montréal. 
Malgré le fait que Côté rapporte que 30 \% de son échantillon de collégiens affirment parler autant le français que l'anglais dans la vie de tous les jours, ce qui est une masse critique à ne pas négliger, peu d'informations sur le rôle de ces «bilingues » ou «multilingues » dans les relations entre francophones et anglophones ne sont encore disponibles, ni dans le contexte d'une scolarisation commune, ni lors d'activités communautaires mixtes ou même de l'environnement social (vie de quartier) ou dans les milieux de travail. Pourtant, dans un contexte comme celui de Montréal, où 18,5\% de la population est allophone et va généralement apprendre l'une des deux langues ou même les deux langues officielles et où près de $3 \%$ de la population déclare avoir plus d'une langue maternelle (Statistique Canada, 2001), il est essentiel d'étudier davantage le rôle de ces «bilingues » et "multilingues » dans les relations entre francophones et anglophones tant dans le milieu communautaire qu' au niveau des institutions postsecondaires.

\section{Le portrait bruxellois}

Pour Bruxelles, ce seront d'une part des possibilités de contacts entre communautés linguistiques au niveau scolaire et communautaire qui seront présentées et d'autre part, l'impact des contacts sur les attitudes des jeunes francophones et néerlandophones.

\section{Possibilités de contacts entre jeunes francophones et néerlandophones à Bruxelles}

Outre les contacts entre élèves francophones et néerlandophones dans les écoles néerlandophones de Bruxelles, Janssens et Van Mensel (2006) ont dégagé d'autres espaces de contacts intercommunautaires dans leur analyse des centres culturels bruxellois financés par la Communauté flamande. Les "gemeenschapscentra" (littéralement, centres communautaires) sont, tout comme l'enseignement néerlandophone, des éléments phares de la politique de présence linguistique des autorités flamandes dans la capitale. Ces centres ont donc une vocation bien plus large que celle d'une offre culturelle de proximité à la minorité néerlandophone. Outre un programme culturel, ils proposent des ateliers, stages et cours dans des domaines aussi divers que le sport, le récréatif pour enfants et adolescents, la musique et la danse, le tout étant proposé en néerlandais. Si les activités pour enfants attirent un public très diversifié du point de vue linguistique et culturel, il faut noter que parmi les jeunes participants "francophones", on compte tant des jeunes de familles francophones, que de familles issues de l'immigration, ces derniers y étant principalement attirés par les cours de hip hop, breakdance ou danse du ventre.

Les autres endroits où jeunes francophones et néerlandophones se côtoient dans la capitale sont les grands espaces de spectacles, comme l'Ancienne Belgique, et le monde associatif tant culturel que sociopolitique. Pour ces derniers, il s'agit d'ailleurs souvent de mouvements de citoyens, qui dépassent les clivages linguistiques traditionnels de Bruxelles. Les rencontres d'associations, comme Amnesty International, ayant lieu dans certains de ces "gemeenschapcentra" néerlandophones, accueillent des sympathisants aussi bien francophones que néerlandophones. 
Enfin, il faut mentionner que la Communauté flamande investit largement dans les activités parascolaires et récréatives, au départ des écoles néerlandophones. Les stages de musique, de sport, et les plaines de jeux organisés en néerlandais pendant les vacances, les mercredis après-midi ou les week-ends drainent un public linguistiquement très hétérogène, où les contacts entre néerlandophones et non-néerlandophones (francophones et/ou allophones) sont favorisés.

Il reste que la quantité et la qualité des contacts intercommunautaires réels, engendrés par ces activités, gravitant autour des centres communautaires et scolaires néerlandophones de Bruxelles, varient fortement d'une commune à l'autre, en fonction de facteurs géographiques et démographiques et sont difficilement mesurables empiriquement.

Cependant, un volet d'une large étude sur les écoles néerlandophones de Bruxelles (Housen, Mettewie, \& Pierrard, 2002; Housen \& Pierrard, 2004; Van Mensel, Housen, Mettewie, \& Pierrard, 2005) a analysé les effets sur les attitudes et la motivation du contact intercommunautaire entre élèves francophones et néerlandophones (Mettewie, 2004). Ne seront présentés ici que les aspects concernant les attitudes envers les langues et les communautés linguistiques en présence. D’autres articles traitent de l'impact du contact intercommunautaire sur la motivation, (Mettewie, 2003; Mettewie, Housen, \& Pierrard, 2004) et sur les compétences linguistiques (Mettewie, Housen, \& Pierrard, 2005; Van Mensel, Pierrard, \& Housen, 2004).

\section{L'impact des contacts intercommunautaires sur les attitudes des jeunes francophones et néerlandophones à Bruxelles}

L'enseignement néerlandophone à Bruxelles accueille une (très) large population d'élèves non néerlandophones (voir plus haut), alors que ces écoles étaient, à l'origine, prévues pour accueillir la minorité linguistique néerlandophone de la capitale. L'enseignement se doit d'y être dispensé uniquement en néerlandais (le néerlandais qui est considéré comme étant la langue maternelle des élèves, même si aujourd'hui la réalité est bien différente). L'hétérogénéité linguistique de la population scolaire a des implications non négligeables au niveau organisationnel et pédagogique (Mettewie, Housen, Pierrard et al., 2005), mais présente l'avantage d'être un lieu de contact entre francophones et néerlandophones. Les conditions des théories de contacts intercommunautaires sont ici en grande partie rassemblées. En effet, (1) l'interaction est fréquente et assez intensive dans les classes, (2) les personnes d'autorité, dans ce cas les parents et les enseignants, soutiennent l'intégration des communautés au sein de l'école (même si certains enseignants sont assez réservés, voire négatifs face à cette hétérogénéité linguistique) et (3) les deux langues et communautés jouissent d'un statut élevé et d'une reconnaissance tant au niveau régional que national (Mettewie, 2004). Quant au niveau de coopération, peu de données empiriques font état d'un manque de coopération entre élèves et/ou de compétition, pouvant nuire aux relations intergroupes.

Afin de mesurer l'impact de la situation de contact sur les attitudes des élèves francophones et néerlandophones dans ces écoles néerlandophones de Bruxelles, Mettewie (2004) les a comparées à celles d'élèves de groupes contrôles en Flandre 
(région néerlandophone) et en Wallonie (région francophone), où les élèves n'ont pas de contacts avec des membres de l'autre communauté linguistique $(\mathrm{N}=686)$. Les comparaisons indiquent que les élèves, âgés de 12, 15 et 18 ans, en situation de contact intercommunautaire, développent des attitudes langagières plus positives que les élèves en Flandre et en Wallonie. C'est le cas pour leurs attitudes envers la langue, la communauté et la culture de l'autre groupe linguistique, mais non pour les attitudes envers leur langue maternelle et leur propre communauté. Ceci suggère que le contact a un effet positif sur la perception de l'autre, mais n'engendre pas d'attitudes négatives (ou moins positives) envers la langue première et la communauté. Cependant des analyses différenciées pour les deux groupes linguistiques en contact dans l'enseignement néerlandophone de Bruxelles montrent que ce contact a un impact significatif sur les attitudes des francophones, mais peu (voire pas) pour les néerlandophones. Ceci signifie que les francophones dans l'enseignement néerlandophone développent des attitudes significativement plus positives envers le néerlandais et les néerlandophones que les élèves en Wallonie. Ce bénéfice du contact pour les élèves francophones par rapport à leurs condisciples néerlandophones, s'explique par le fait que ceux-ci se trouvent dans un contexte de contact bien plus intensif et structuré. En effet, pour les élèves néerlandophones dans les écoles néerlandophones le contact avec le français se limite au cours de français langue étrangère et le contact avec les francophones d'une part, et à la cour de récréation d'autre part. Les francophones des écoles néerlandophones, quant à eux, multiplient les contacts tant avec la langue néerlandaise, qu'avec les locuteurs (les élèves néerlandophones et les enseignants) dans toutes les activités scolaires, sauf peut-être dans les cours de récréation, où, malgré une politique stricte d'emploi du néerlandais, le français est néanmoins parlé. De plus, il faut noter que deux tiers de ces francophones disent utiliser le néerlandais (avec ou sans français) avec leur groupe d'amis et/ou lors d'activités extrascolaires. Quant aux élèves néerlandophones, seul un cinquième d'entre eux déclarent utiliser la langue de l'autre communauté avec leurs amis et/ou lors d'activités récréatives ou sportives et ce toujours en combinaison avec leur langue maternelle. L'usage du néerlandais par ces élèves francophones ne se limite donc pas à l'école néerlandophone.

Enfin, les analyses des attitudes langagières des élèves dans l'enseignement néerlandophone de Bruxelles ont également mis en lumière le profil particulier que présentent les élèves qui sont bilingues néerlandais/français de naissance, représentant près de $20 \%$ de la population scolaire (Mettewie, 2004). Même si ces bilingues affichent des attitudes légèrement plus positives envers le français, qui est la langue dominante à Bruxelles, les analyses (repeated measures) montrent qu'ils ont des attitudes tout aussi positives envers les deux communautés et ne semblent pas faire de différences entre celles-ci contrairement aux autres élèves, même ceux en situation de contact intensif comme c'est le cas des francophones dans l'enseignement néerlandophone (Mettewie, 2004). En outre, tout en restant extrêmement positifs envers les communautés, ils apparaissent moins chauvinistes que les élèves issus d'une seule communauté linguistique. Le profil de médiateur entre deux communautés de ces bilingues mériterait sans doute d'être étudié plus en détail. 
Sur la base de ces résultats, il apparaît que la situation particulière de contact intercommunautaire dans les écoles néerlandophones de Bruxelles a un impact positif sur les attitudes, principalement des francophones, envers la langue et la culture de l'autre communauté. Ce contact est source d'ouverture et d'appréciation réciproque, qui est précieuse dans un contexte de polarisation de plus en plus sensible entre les communautés linguistiques majoritaires en Belgique.

Cependant, il est important de noter que même si les possibilités de contacts entre communautés linguistiques à Bruxelles semblent nombreuses, elles n'en sont pas moins trompeuses. En effet, que ces contacts se situent dans le cadre des écoles néerlandophones ou dans le cadre associatif, culturel et récréatif des centres communautaires néerlandophones de la capitale, ceux-ci restent quantitativement un phénomène marginal (Janssens, 2001; 2007, Janssens \& Van Mensel 2006, Mettewie, 2004) dans une ville officiellement bilingue.

\section{Discussion et conclusion}

À la lecture de ces résultats de recherche, il apparaît que les expériences de contacts entre communautés linguistiques de Montréal et de Bruxelles sont positives, tant dans les milieux scolaires, lorsqu'elles sont possibles, que dans les milieux communautaires. Si ces deux contextes sont différents à bien des égards, ils convergent toutefois à plusieurs niveaux. Ainsi trois constats s'imposent lors de la mise en parallèle des situations montréalaise et bruxelloise.

Premièrement, il apparaît que les différents milieux observés, tant du côté montréalais que bruxellois, mettent en place les conditions essentielles au bon déroulement des rapports intercommunautaires, selon la théorie des contacts. Deuxièmement, nous observons également, dans ces deux contextes de contacts interlinguistiques, qu'il y a un lien clair entre la quantité de contacts qu'ont les jeunes (que ce soit dans le milieu scolaire ou communautaire) et leurs attitudes positives à l'égard de la langue et des membres de «l'autre » groupe linguistique. Finalement, nous constatons que la proximité avec l'autre groupe linguistique que les jeunes développent lors des contacts n'engendre pas pour autant une distance face à la langue maternelle ou face à la communauté linguistique d'origine. Il semble donc qu'avoir des contacts intercommunautaires n'induise pas une assimilation avec «l'autre » groupe linguistique.

De plus, du bilan que nous avons présenté émergent plusieurs questions qui devraient faire l'objet de recherches en interface Bruxelles / Montréal, qui soient à la fois complémentaires et comparatives. Trois axes de recherche se dégagent de l'analyse des situations intercommunautaires montréalaise et bruxelloise.

Premièrement, il nous paraît essentiel de poursuivre des recherches concernant les liens entre compétences linguistiques (mesurées et/ou auto-évaluées), attitudes et contacts intergroupes, afin de dégager une direction dans les relations entre les variables dans l'étude des contacts. Ceci implique un approfondissement des données concernant les compétences linguistiques (pourquoi certains jeunes se sentent 
moins compétents en langue seconde, quel impact cela a-t-il, quels niveaux de compétences linguistiques vont de pair avec les attitudes les plus positives ou négatives?) et l'utilisation complémentaire de protocoles longitudinaux et/ou qualitatifs. Outre la direction causes/effets dans les variables tant contextuelles qu'individuelles et linguistiques, ces analyses permettraient de dégager des pistes pour contrer des impacts négatifs dans les relations intercommunautaires et linguistiques.

Deuxièmement, une comparaison de l'impact des contacts dans le cadre des enseignements de langue seconde s'impose entre d'une part des situations d'apprentissage institutionnalisées, de type classes d'immersion et des échanges structurés et encadrés didactiquement, et d'autre part des situations non institutionnalisées, comme c'est le cas dans l'enseignement néerlandophone à Bruxelles, ou les collèges anglophones de Montréal. Se pose ainsi la question de savoir si l'enseignement en immersion (avec un contact intercommunautaire limité aux enseignants) permet de développer des attitudes aussi positives envers la langue seconde et ses locuteurs que la situation de contacts non institutionnalisés entre jeunes. S'appuyant sur l'expérience positive du milieu communautaire de Montréal, du milieu scolaire bruxellois et du milieu collégial anglophone du Québec, les écoles primaires et secondaires, francophones et anglophones ou néerlandophones semblent avoir avantage à favoriser entre elles les jumelages récurrents et «interactifs en faceà-face», tant pour atteindre des objectifs d'apprentissage linguistique que pour l'impact social (rapprochement) qui découle de ces contacts. Reste à analyser quels sont les leviers qui permettent la mise en place de tels échanges avec des conditions de contact favorables à l'amélioration des rapports intergroupes et l'impact réel de ces jumelages «institutionnalisés» et de contact non spontané sur les attitudes des jeunes.

Troisièmement, les comparaisons ont déjà clairement montré que, dans les deux métropoles, les rapports intergroupes dépassent largement les deux communautés linguistiques traditionnelles. En effet, ces contacts intercommunautaires incluent inévitablement des rapports avec des bilingues issus des deux communautés historiques et avec d'autres communautés linguistiques issues de l'immigration. Ces contacts s'avèrent d'autant plus complexes qu'une masse critique de Montréalais et de Bruxellois sont porteurs d'une forme plus ou moins développée de bilinguisme voire de multilinguisme. Dès lors, il semble incontournable d'analyser d'un côté de manière comparative les formes de bilinguisme et de multilinguisme que l'on retrouve dans ces deux contextes, tant au niveau de l'identité que des usages linguistiques ${ }^{4}$, et d'un autre côté, le rôle des bilingues (natifs ou tardifs) et des multilingues dans le rapport et/ou le rapprochement des communautés linguistiques, dans un contexte de polarisation.

Dans cette contribution, nous avons tenté de montrer les convergences entre les contextes montréalais et bruxellois dans le cadre des relations intercommunautaires, tant pour les projets déjà réalisés que pour les trois axes de recherche à

4. Deux études sur des aspects de cette thématique sont en cours (Janssens, 2007 et Côté, à paraître en 2008). 
venir. Ainsi, Montréal et Bruxelles s'avèrent être des terrains de recherche idéaux pour étudier et comparer tant la dimension linguistique des contacts intergroupes que le rôle du bi- ou multilinguisme et des bilingues dans ces rapports, et ainsi contribuer à l'enrichissement des théories classiques expliquant les relations intergroupes.

\section{Références bibliographiques}

BREWER, M. B., et Miller, N. (1996). Intergroup contact, cooperation, and competition: Does togetherness make friends? Intergroup Relations. Buckingham: Open University Press p. 107-133.

CLÉMENT, R., Noels, K. et Denault, B. (2001). Interethnic contact, identity, and psychological adjustment: The mediating and moderating roles of communication. Journal of Social Issues, 57 (3), p. 559-577.

CÔTÉ, B. (2005). Étude des rapports entre jeunes "francophones » et "anglophones " dans des collèges anglophones $d u$ Québec. Thèse de doctorat non publiée, Université de Montréal, 230 p.

CÔTÉ, B. (2007). Évaluation de trois formes d'un programme de jumelage entre des écoles primaires francophones et des écoles primaires anglophones de la région de Montréal. Projet de recherche pour le Ministère de l'Éducation, des Loisirs et du Sport du Québec, soumis au Comité d'Éthique de l'Université de Sherbrooke.

DOVIDIO J., Gaertner, S., et Kawakami, K. (2003). Intergroup contact: The past, present, and the future. Group Processes and Intergroup Relations, 6 (1), p. 5-21.

GAERTNER, S.L., Rust, M.C., Dovidio, J. F., Bachman, B. A. et Anastasio, P. A. (1994). The contact hypothesis: The role of a commun ingroup identity on reducing intergroup bias. Small Group Reasearch, 25 (2), p. 224-249.

HEWSTONE, M., et Brown, R. (1986). Contact and conflict in intergroup encounters. New York: Basil Blackwell.

HOUSEN, A., Mettewie, L., et Pierrard, M. (2002). Rapport Beleidsgericht onderzoek PBO/98/2/36. Taalvaardigheid en attitudes van Nederlandstalige en Franstalige leerlingen in het secundair onderwijs in Brussel (Research report). Brussel: Centrum voor Linguïstiek - Vrije Universiteit Brussel.

HOUSEN, A., et Pierrard, M. (2004). Meertaligheid in Nederlandstalig secundair onderwijs in Brussel: verslag van een effectenstudie, dans A. Housen, M. Pierrard et P. Van de Craen (Éds.), Brusselse Thema's. Taal, Attitude en Onderwijs in Brussel. Brussel: VUBPress, p. 9-33. 
ISLAM, M.R. et Hewstone, M. (1993). Dimensions of contact as predictors of intergroup anxiety, perceived out-group variability and out-group attitude : an integrative model. Personality and Social Psychology Bulletin, 19, p. 700-710.

JANSSENS, R. (2001). Taalgebruik in Brussel: Taalverhoudingen, taalverschuivingen en taalidentiteit in een meertalig Brussel. Brussel: VUBPress.

JANSSENS, R. (2007). Van Brussel gesproken. Taalgebruik, taalverschuivingen en taalidentiteit in het Brussels Hoofdstedelijk Gewest. Brussel: VUBPress.

JANSSENS, R., et Van Mensel, L. (2006). Publieksonderzoek Gemeenschapscentra. Een onderzoek naar het profiel van de bezoekers van de Vlaamse gemeenschapscentra in Brussel (Research report). Brussel: Vrije Universiteit Brussel.

MCANDREW, M., et Gagnon, F. (2000). Relations ethniques et éducations dans les sociétés divisées. Montréal: L’Harmattan.

METTEWIE, L. (2003). Contacthypothese en taalleermotivatie in Nederlandstalige scholen in Brussel. Toegepaste taalwetenschap in artikelen, 70 (2), p. 79-89.

METTEWIE, L. (2004). Attitudes en motivatie van taalleerders in België. Een sociaal-psychologisch onderzoek naar het verwerven van de eerste en tweede taal door Nederlandstalige, Franstalige en tweetalige leerlingen in het secundair onderwijs in Brussel (Thèse de doctorat). Brussel: Vrije Universiteit Brussel.

METTEWIE, L., Housen, A., et Pierrard, M. (2004). Invloed van contact op taalattitudes en taalleermotivatie in het Nederlandstalig onderwijs in Brussel, dans A. Housen, M. Pierrard et P. Van de Craen (Éds.), Brusselse Thema's. Taal, Attitude en Onderwijs in Brussel. Brussel: VUBPress, p. 35-65.

METTEWIE, L., Housen, A., et Pierrard, M. (2005). Les élèves francophones dans l'enseignement néerlandophone à Bruxelles deviennent-ils bilingues français/néerlandais? dans P. Hiligsmann et e.a. (Éds.), Neerlandistiek in Frankrijk en in Franstalig België / Les études néerlandaises en France et en Belgique francophone. Louvain-la-Neuve, 15-17 maart/mars 2004. Louvain-laNeuve: Faculté de Philosophie et Lettres/Academia-Bruylant, p. 165-176.

METTEWIE, L., Housen, A., Pierrard, M., et Van Mensel, L. (2005). Franstalige leerlingen in het Nederlandstalig onderwijs in Brussel: verslag van een effectenstudie. School en samenleving, 8 (87), p. 105-125.

METTEWIE, L., Van Mensel, L., et Belang, D. (2006). Entreprises bruxelloises et langues étrangères. Pratique et coût d'une main d'œuvre ne maîtrisant pas les langues étrangères. Bruxelles: TIBEM - Ministère de l'Economie et de l'Emploi de la Région de Bruxelles-Capitale.

METTEWIE, L. (2008). Elèves non-néerlandophones dans l'enseignement néerlandophone à Bruxelles: analyse des répercussions éducatives de la traversée de la frontière linguistique, dans L. Puren et S. Babault (Éds.), L'éducation au-delà des frontières. Paris: L'Harmattan. 
PAGÉ, M., Côté, B. et Lasry (2007). Les contacts entre jeunes francophones et anglophones dans l'Île de Montréal. Rapport de recherche non publié, CEETUM, $24 \mathrm{p}$.

PETTIGREW, T.F. (1998). Intergroup contact theory. Annual Review of Psychology, 49, p. 65-85.

STEPHAN, W.J., et Stephan, C. W. (1985). Intergroup anxiety. Journal of Social Issues, 41 (3), p. 157-175.

TAYLOR, D.M., Dubé, L., et Bellerose, J. (1986). Intergroup Contact in Québec: Myth or Reality? dans M. Hewstone et R. Brown, Contact and conflict in intergroup encounters. New York: Basil Blackwell, p. 93-106.

VAN MENSEL, L., Housen, A., Mettewie, L., et Pierrard, M. (2005). Multilinguisme dans l'enseignement néerlandophone à Bruxelles: résultats d'une étude pilote, dans E. Witte, L. Van Mensel, M. Pierrard, L. Mettewie, A. Housen et R. De Groof (Éds.), Language, Attitudes et Education in Multilingual Cities. Brussel: Koninklijke Vlaamse Academie van België voor Wetenschappen en Kunsten, p. 133-142.

VAN MENSEL, L., Pierrard, M., et Housen, A. (2004). Taalvaardigheid van Nederlandstalige en Franstalige leerlingen in het Nederlandstalig secundair onderwijs in Brussel, dans A. Housen, M. Pierrard et P. Van de Craen (Éds.), Brusselse Thema's. Taal, Attitude en Onderwijs in Brussel. Brussel: VBUBPress, vol. 12, p. 67-110.

WILDER, D.A., et Shapiro, P.N. (1989). Role of competition-induced anxiety in limiting the beneficial impact of positive behavior by an out-group member. Journal of Personality and Social Psychology, 56 (1), p. 60-69.

\section{Sites Internet:}

http://cansim2.statcan.ca/cgi-win/cnsmcgi.pgm?Lang=F\&SP_Action=Result\&SP_ ID $=50004 \& S P \_T Y P=5 \& S P \_S o r t=1$

http://im.metropolis.net/research-policy/research_content/atlas/carteA2.html (Archambault, Ray, Rose et Séguin, consulté le $1^{\text {er }}$ octobre 2007).

http://statbel.fgov.be/figures/d21_fr.asp\#3 (consulté le 1 ${ }^{\mathrm{er}}$ octobre 2007).

http://www.clilcompendium.com/

http://www.enseignement.be/citoyens/thematiques.asp

http://www.euroclic.net/

http://www.senate.be/www/?MIval=/index_senate\&MENUID=22000\&LANG=fr

(consulté le $1^{\text {er }}$ octobre 2007).

http://www.vgc.be/Onderwijs/ 